decompensation, permitting them to implement interventions that might avert hospitalization.

Original article Chaudhry SI et al. (2007) Patterns of weight change preceding hospitalization for heart failure. Circulation 116: $1549-1554$

\section{Effectiveness of ACE inhibition in patients with Marfan's syndrome on $\beta$-blockade}

The standard treatment for the connective tissue disorder Marfan's syndrome (MFS) is $\beta$-blockade, aimed at reducing aortic wall stress. Angiotensin II type 1 and 2 receptors $\left(A T_{1}\right.$ and $\left.A T_{2}\right)$ are implicated in the pathogenesis of MFS. Researchers have tested the efficacy of the angiotensin-converting-enzyme (ACE) inhibitor perindopril at reducing aortic stiffening and dilatation-two of the most serious complications of MFS - and found it to have additive benefit in patients receiving standard $\beta$-blocker therapy.

Over a period of 24 weeks, 10 patients with MFS received $8 \mathrm{mg}$ perindopril daily, and 7 patients received placebo. Patients on perindopril had significantly reduced arterial stiffness (measured as increased systemic arterial compliance and reduced central and peripheral pulse wave velocities). Systolic and end-diastolic aortic diameters were significantly lower with the drug than with placebo. Perindopril reduced the mean arterial pressure, but the changes in stiffness and aortic diameters were still significant when this factor was included as a covariate in multivariate analyses.

The authors also studied the relationship between the aortic changes and plasma levels of matrix metalloproteinase (MMP) and transforming growth factor- $\beta$ (TGF- $\beta$ ) - two factors that are partly responsible for the aortic degeneration in MFS. MMP-2, MMP-3 and TGF- $\beta$ were reduced significantly with perindopril compared with placebo. The reductions in aortic stiffness and aortic root diameter with perindopril could, therefore, result from reduced $A T_{1}$ and $A T_{2}$ receptor signaling; reduced $A T_{1}$ leads to reduction in MMP-2, MMP-3 and TGF- $\beta$ signaling, while reduced $A T_{2}$ could protect from cystic medial degeneration.

Original article Ahimastos AA et al. (2007) Effect of perindopril on large artery stiffness and aortic root diameter in patients with Marfan syndrome: a randomized controlled trial. JAMA 298: 1539-1547

\section{Sirolimus-eluting stents can be used in patients with diabetes and coronary artery disease}

Patients with diabetes mellitus and coronary artery disease (CAD) are at high risk of adverse cardiovascular events. They are also more likely to suffer complications during invasive therapeutic procedures, such as percutaneous treatment for coronary stenosis and stent implantation. In this study, Baumgart et al. investigated the use of sirolimus-eluting stents (SESs) in such patients to assess impact on neointimal proliferation, in-stent restenosis rates, and morbidity and mortality within 1 year of stent placement.

The multicenter study involved 200 patients with diabetes and CAD; 98 received SESs and 102 were given bare-metal stents (BMSs). Analysis of the depth of in-segment late luminal loss 8 months after stent implantation revealed that patients with SESs lost an average of $0.18 \mathrm{~mm}$ of luminal tissue at the stent site. This amount was significantly less than that lost in patients who received BMSs, in whom an average of $0.74 \mathrm{~mm}$ was lost. Rates of restenosis were lower in the SES group than in the BMS group ( $8.8 \%$ vs $42.1 \%$ ). At 12 months, only $14.7 \%$ of patients in the SES group had experienced a major cardiac event, compared with $35.8 \%$ of patients in the BMS group. Overall mortality was similar in the two groups.

The authors conclude that the SES is an attractive treatment option for patients with diabetes and CAD, being associated with a significantly lower risk of major cardiac events and lower in-segment late luminal loss than the BMS.

Original article Baumgart D et al. (2007) One-year results of the SCORPIUS study: a German multicenter investigation on the effectiveness of sirolimus-eluting stents in diabetic patients. J Am Coll Cardiol 50: 1627-1634

\section{New survey reveals global extent of obesity crisis}

The results of IDEA - the International Day for Evaluation of Abdominal Obesity-provide new insights into worldwide patterns of obesity. Conducted in 2005, the study comprised 69,409 men and 98,750 women from 63 countries, recruited and assessed at primary care facilities using standardized protocols. 